Bio - grafia. Escritos sobre la Biología y su Enseñanza. ISSN 2027

Edición Extraordinaria. p.p. 317 - 326

Memorias del VIII Encuentro Nacional de Experiencias en Enseñanza de la Biología y la Educación Ambiental. III Congreso Nacional de Investigación en Enseñanza de la Biología.

\title{
FORTALECIMIENTO DE LA CULTURA AMBIENTAL POR PARTE DE LOS ESTUDIANTES DE NOVENO GRADO DE LA INSTITUCIÓN EDUCATIVA MARÍA CRISTINA ARANGO DE PASTRANA DE LA CIUDAD DE NEIVA, HUILA
}

Strengthening environmental culture by the ninth graders of School Maria Cristina de Pastrana Arango city Neiva

Dora Magaly García Ibarra, Universidad Surcolombiana Jose Fernando Ramírez Bermúdez, Universidad Pedagógica y Tecnológica de Colombia

\section{RESUMEN}

El presente estudio tuvo como objetivo fortalecer la cultura ambiental por medio de actividades de concientización y embellecimiento de la institución educativa María Cristina Arango de Pastrana de la Ciudad de Neiva, Huila. La investigación se desarrolló desde un enfoque cualitativo - descriptivo empleando como método el análisis de contenido, haciendo uso del cuestionario, observación participante y la entrevista con un grupo de 28 estudiantes de grado noveno. En cuanto a la metodología llevada a cabo, inicialmente se desarrolló una revisión y análisis del Proyecto Ambiental Escolar (PRAE), a través de la aplicación de una matriz de análisis categorial de fortalezas y debilidades en la estructura del documento. Posteriormente, se realizaron jornadas de limpieza, carteleras, siembras y charlas informativas, en las cuales los estudiantes concientizaron a la comunidad educativa acerca del cuidado del ambiente. Y Finalmente, se aplicaron entrevistas y cuestionarios a los participantes para analizar las representaciones ambientales de la institución educativa. En la investigación se evidenció que el PRAE no tiene dentro de su organización los parámetros establecidos por la política nacional de educación ambiental; las representaciones ambientales emergentes de los instrumentos aplicados infieren una cultura ambiental vista desde el cuidado, conservación y preservación del ambiente. Sin embargo, las actividades de concientización y embellecimiento incitaron al buen uso de los recursos naturales, desarrollando el sentido de pertenencia por la Institución Educativa y en modo general al cuidado de muestro planeta Tierra.

\section{PALABRAS CLAVE}

Cultura ambiental, representación ambiental, Proyecto Ambiental Escolar. 
Bio - grafia. Escritos sobre la Biología y su Enseñanza. ISSN 2027

Edición Extraordinaria. p.p. 317 - 326

Memorias del VIII Encuentro Nacional de Experiencias en Enseñanza de la Biología y la Educación Ambiental. III Congreso Nacional de Investigación en Enseñanza de la Biología.

\begin{abstract}
This study aimed to strengthen environmental culture through awareness activities and beautification of the school Maria Cristina Pastrana Arango of the City of Neiva, Huila. The research was developed from a qualitative approach - using as descriptive content analysis method, using the questionnaire, participant observation and interview with a group of 28 ninth graders. In terms of methodology it carried out a review and analysis initially School Environmental Project (RESP) was developed through the application of a matrix of categorical analysis of strengths and weaknesses in the structure of the document. Subsequently, cleanups, billboards, plantings and information sessions, in which students concientizaron the educational community about the care of the environment is made. And finally, interviews and questionnaires were applied to the participants to analyze the environmental performances of the school. In the investigation it became clear that the RESP does not have within their organization the parameters established by the National Environmental Education Policy; emerging environmental performances of the instruments applied infer an environmental culture seen from the care, conservation and preservation of the environment. However, awareness activities and beautification prompted the good use of natural resources, developing a sense of belonging to the educational institution and generally show the care of planet Earth.
\end{abstract}

\title{
KEYWORDS
}

Environmental culture, beautification, Institutional environmental project.

\section{INTRODUCCIÓN}

En nuestra profesión docente es importante tener una visión más holística de las realidades, que seamos capaces de comprender, utilizar, contextualizar y promover saberes, habilidades, y destrezas relacionados con la ciencias naturales. Es por ello que la situación que enmarca un educador en ciencias naturales es que tenga la capacidad de hacer lectura de la realidad buscando desde sus clases solucionar problemas que nos rodean los cuales sean indispensables para comprender los fenómenos y a contribuir al desarrollo, sostenimiento y conservación del ambiente.

Las relaciones hombre - sociedad - naturaleza determinan la orientación de la dimensión ambiental de la actividad humana, expresada en las políticas de los estados, de la sociedad en su componente comportamental y ambiental. Según Bayon, et al (2006) "A través de la educación el hombre interioriza la cultura; el hombre, en tanto sujeto educable, es capaz 
Bio - grafia. Escritos sobre la Biología y su Enseñanza. ISSN 2027

Edición Extraordinaria. p.p. 317 - 326

Memorias del VIII Encuentro Nacional de Experiencias en Enseñanza de la Biología y la Educación Ambiental. III Congreso Nacional de Investigación en Enseñanza de la Biología.

de construir y producir conocimientos, de desarrollar su capacidad y formar y reorientar sus valores", lo que hace posible que las personas modifiquen sus conducta a través de su actividad social, para contribuir como sujeto individual a la transformación de la realidad.

Según Reigota (1990), "El medio ambiente no es un concepto científico, ya que éstos son entendidos y empleados universalmente como tales. Por su carácter variado y difuso considera la noción de medio ambiente como una representación social y resalta que el primer paso para la realización de la educación ambiental consiste en la identificación de las representaciones que tienen las personas involucradas en el proceso educativo".

\section{METODOLOGÍA}

La investigación se ubica desde un paradigma interpretativo, con un enfoque cualitativodescriptivo, donde se plantea que "el investigador debe construir una imagen compleja, holística, y detallada de las perspectivas de los informantes, conduciendo el estudio en una situación natural que describa, interprete y analice la estructura del PRAE en relación con las representaciones ambientales que tienen los estudiantes en torno a la cultura ambiental" Vasilachis (2006).

El estudio se llevó a cabo con 28 estudiantes de grado noveno de la institución Educativa María Cristina Arango de Pastrana de la Ciudad de Neiva, Huila. Inicialmente se analizó el PRAE con base a una matriz de análisis categorial, luego se diseñó y aplicó un cuestionario inicial para conocer los aspectos relevantes que los estudiantes tienen sobre la cultura ambiental. Posteriormente, se procedió a desarrollar una actividad de carácter ambiental con el propósito que los estudiantes identificaran aspectos relevantes relacionados con el ambiente, como su definición, características, problemáticas, fortalezas, aportes sociales y el diseño de estrategias para el fortalecimiento de la cultura ambiental en la institución. Enseguida, se realizó una jornada ambiental de embellecimiento y ornamentación que aportara a la consolidación del sentido de pertenencia por la institución Educativa. Finalmente, se aplicó nuevamente la entrevista para contrastar los cambios conceptuales, metodológicos y actitudinales en torno a la cultura ambiental.

La investigación se desarrolló desde un enfoque cualitativo el cual tiene la característica principal de recolectar los datos sin necesidad de que exista una medición numérica, de tal manera que se utiliza en primera instancia para descubrir y refinar preguntas de investigación. Así mismo, se recogen perspectivas obtenidas de los participantes a través de experiencias individuales y colectivas. 
Bio - grafia. Escritos sobre la Biología y su Enseñanza. ISSN 2027

Edición Extraordinaria. p.p. 317 - 326

Memorias del VIII Encuentro Nacional de Experiencias en Enseñanza de la Biología y la Educación Ambiental. III Congreso Nacional de Investigación en Enseñanza de la Biología.

Además, las investigaciones de tipo cualitativo se basan en un proceso en el que se explora y se describe para luego poder generar perspectivas teóricas, siendo prácticas interpretativas que plasman al mundo en documentos, grabaciones o simplemente observaciones. (Hernández, Fernández \& Baptista, 2006).

Por su parte, el método utilizado fue el análisis de contenido el cual corresponde a "un proceso en el que se significa el contenido de un texto o un documento de acuerdo a los objetivos planteados en la investigación" Bardín (1987) citado en López (2002). Por tanto, se utilizó el software para el análisis cualitativo de datos ATLAS ti, versión 7.0.

Una de las técnica de recolección de información utilizada fue la de cuestionarios, que según Martín (2004), "es un instrumento para la recolección de información, diseñado para cuantificarla y universalizarla", ya que antes de proceder a medir algo debemos tener una idea muy clara de lo que queremos decir o sea definir el constructo

\section{RESULTADOS Y DISCUSIÓN}

En un primer momento, se presentara los resultados sobre el análisis del PRAE, y en segunda instancia, se relacionara las representaciones ambientales iniciales de los estudiantes en torno a la cultura ambiental.

\section{Análisis categorial de PRAE.}

Se consideraron cinco categorías centrales de la investigación, las cuales se elaboraron teniendo como base la fundamentación de la política Nacional de educación ambiental y que aplica para la educación formal que "permiten abarcar el proceso de análisis sobre los saberes y prácticas de la educación ambiental desde cada uno de los estamentos propuestos" (Flórez, 2012). Para determinar el nivel de desarrollo de cada subcategoría se elaboró la siguiente clasificación: 
Bio - grafia. Escritos sobre la Biología y su Enseñanza. ISSN 2027

Edición Extraordinaria. p.p. 317 - 326

Memorias del VIII Encuentro Nacional de Experiencias en Enseñanza de la Biología y la Educación Ambiental. III Congreso Nacional de Investigación en Enseñanza de la Biología.

Tabla 1. Niveles de desarrollo PRAE

\begin{tabular}{|c|l|}
\hline NIVEL & \multicolumn{1}{|c|}{ DESCRIPCIÓN } \\
\hline 0 & AUSENTE \\
\hline 1 & INCOMPLETO \\
\hline 2 & PARCIALMENTE COMPLETO \\
\hline 3 & COMPLETO \\
\hline
\end{tabular}

Fuente: Flórez (2012)

Como referente metodológico para el análisis del PRAE se tomó la matriz de análisis categorial propuesta por Flórez (2012).

Tabla 2. Matriz de Análisis Categorial y resultados

\begin{tabular}{|c|c|c|}
\hline Categoría & subcategoría & PRAE \\
\hline \multirow{2}{*}{$\begin{array}{l}\text { Contexto de a } \\
\text { educación ambiental }\end{array}$} & Referentes nacionales e internacionales & 1 \\
\hline & Concepto de educación ambiental & 2 \\
\hline \multirow[t]{3}{*}{ Sistema Ambiental } & Visión sistémica del ambiente & 1 \\
\hline & Situación ambiental y problemática ambiental & 2 \\
\hline & Lectura de contexto & 1 \\
\hline \multirow{4}{*}{$\begin{array}{l}\text { Currículo y } \\
\text { transversalidad de } \\
\text { la dimensión } \\
\text { ambiental }\end{array}$} & Pedagogía y didáctica de la educación Ambiental & 0 \\
\hline & Contenidos de la educación Ambiental & 1 \\
\hline & La EA, la escuela y el entorno & 0 \\
\hline & Interdisciplinar-dialogo de saberes & 0 \\
\hline \multirow{2}{*}{$\begin{array}{l}\text { Proyecto ambiental } \\
\text { escolar }\end{array}$} & Conceptualización de PRAE & 0 \\
\hline & $\begin{array}{l}\text { Regionalización, concertación, cogestión, e } \\
\text { interculturalidad. }\end{array}$ & 0 \\
\hline
\end{tabular}


Bio - grafia. Escritos sobre la Biología y su Enseñanza. ISSN 2027

Edición Extraordinaria. p.p. 317 - 326

Memorias del VIII Encuentro Nacional de Experiencias en Enseñanza de la Biología y la Educación Ambiental. III Congreso Nacional de Investigación en Enseñanza de la Biología.

\begin{tabular}{|l|l|c|}
\hline & Etapas del desarrollo del PRAE & 0 \\
\hline $\begin{array}{l}\text { Procesos de } \\
\text { seguimiento } \\
\text { evaluación de la } \\
\text { educación ambiental }\end{array}$ & Apoyo y proyección de la educación Ambiental & 0 \\
\cline { 2 - 3 } & Empacto del PRAE & 0 \\
\hline
\end{tabular}

Fuente: Flórez (2012)

De acuerdo a la tabla 2, el documento PRAE presenta falencias en cada una de las subcategorías analizadas, es de resaltar que en la categoría de concepto de educación ambiental el documento tiene un referente que hace alusión a un visión ecológica olvidando lo cultural, lo social y en los demás aspectos que hacen parte del ambiente. En cuanto a la problemática ambiental el escrito platea varias problemáticas pero no es claro el mecanismo por el cual llegaron a esa conclusión. Sin duda alguna, aunque es una primera mirada al estado de la educación ambiental en esta institución, es evidente que falta generar estrategias que convoquen a la dinamización de la educación ambiental en el contexto donde se encuentra la institución.

\section{Representaciones ambientales iniciales}

Los hallazgos permitieron establecer cuatro subcategorías sobre las representaciones de los estudiantes acerca de la Cultura Ambiental: Importancia, Definición y Estrategia (ver figura 1). A continuación presentamos las características de cada una de ellas, así como su frecuencia y algunas evidencias textuales.

Figura 1. Subcategorías de Cultura Ambiental en el cuestionario inicial.

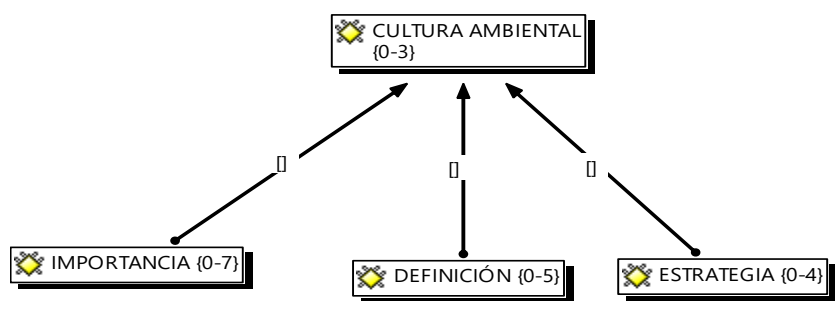

Importancia: Se presentan cinco tendencias (ver figura 2), donde encontramos que la cultura ambiental sirve principalmente para la conservación, preservación y mantenimiento del medio ambiente. Además, para aportarnos conocimiento en el cuidado de las plantas y demás recursos naturales. 
Bio - grafia. Escritos sobre la Biología y su Enseñanza. ISSN 2027

Edición Extraordinaria. p.p. 317 - 326

Memorias del VIII Encuentro Nacional de Experiencias en Enseñanza de la Biología y la Educación Ambiental. III Congreso Nacional de Investigación en Enseñanza de la Biología.

Figura 2. Concepciones acerca de la Importancia de la cultura ambiental en el cuestionario inicial.

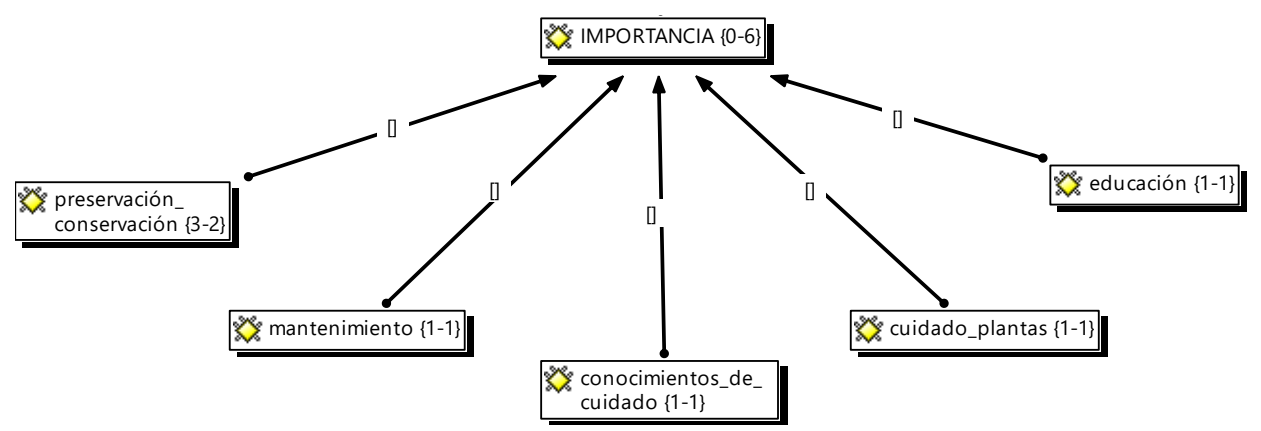

- Tendencia preservación- conservación: En esta tendencia, tres grupos (50\%), consideraron que la cultura ambiental sirve para preservar y conservar la naturaleza.

G1.C1. "La cultura ambiental sirve para la conservación y cuidado de éste".

- Tendencia mantenimiento: Un grupo (16,6\%), contempló que la cultura ambiental es necesaria para el mantenimiento del medio ambiente, infiriendo que los estudiantes excluyen a la capa de ozono de éste extenso grupo.

G4.C1. [Respondiendo a la pregunta ¿Para qué sirve la cultura ambiental?] Sirve para el mantenimiento del medio ambiente y capa de ozono.

Definición: Se presentan cuatro tendencias (ver figura 3), donde se encontró que la cultura ambiental, según los estudiantes, es una campaña de prevención y conservación del medio ambiente. Además, otro estudiante lo relaciona con un grupo de personas que hacen actividades ambientales.

Figura 3. Concepciones acerca de la Definición de la cultura ambiental en el cuestionario inicial. 
Bio - grafia. Escritos sobre la Biología y su Enseñanza. ISSN 2027

Edición Extraordinaria. p.p. 317 - 326

Memorias del VIII Encuentro Nacional de Experiencias en Enseñanza de la Biología y la Educación Ambiental. III Congreso Nacional de Investigación en Enseñanza de la Biología.

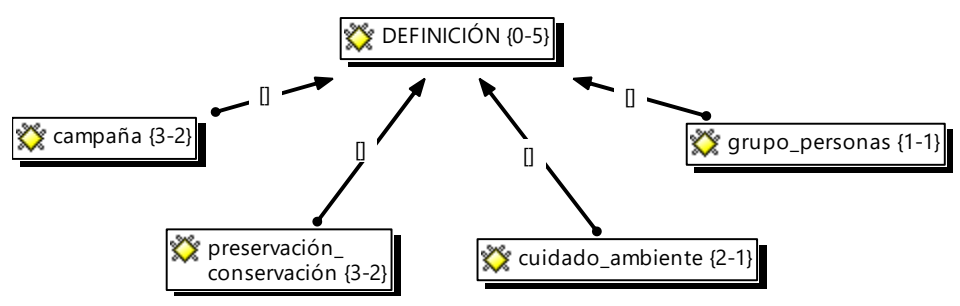

- Tendencia campaña: En esta tendencia, tres grupos (50\%), consideraron que la cultura ambiental es una campaña que se desarrolla en el colegio, la cual tiene como objetivo cuidar el medio ambiente.

G3.C1 [Respondiendo a la pregunta ¿Qué es la cultura ambiental?] "La cultura ambiental en nuestro colegio es una campaña de concientización de los estudiantes cuidar la naturaleza".

- Tendencia preservación-conservación: En esta tendencia, tres grupos (50\%), consideraron que la cultura ambiental es saber cómo preservar y conservar la naturaleza.

G1.C1 [Respondiendo a la pregunta ¿Qué es la cultura ambiental?] "La cultura ambiental es saber cómo preservar la naturaleza de diferentes tipos de entornos".

Estrategia: Se presentan tres tendencias (ver figura 4), donde los estudiantes reconocen diferentes tipos de estrategias para el fortalecimiento de la cultura ambiental en la institución, enfatizando principalmente actividades como no botar basura, ahorrar agua, reciclaje, y campañas.

Figura 4. Concepciones acerca de la estrategia para la cultura ambiental en el cuestionario inicial.

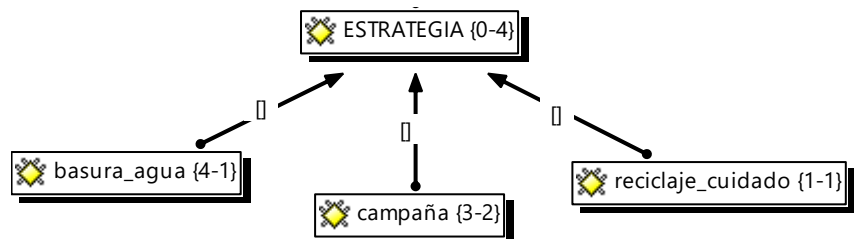

- Tendencia basura-agua: En esta tendencia cuatro grupos (66,6\%), reconocieron que el hecho de no botar basura y ahorrar agua sirven como estrategia para el fortalecimiento de la cultura ambiental en la institución. 
Bio - grafia. Escritos sobre la Biología y su Enseñanza. ISSN 2027

Edición Extraordinaria. p.p. 317 - 326

Memorias del VIII Encuentro Nacional de Experiencias en Enseñanza de la Biología y la Educación Ambiental. III Congreso Nacional de Investigación en Enseñanza de la Biología.

G5.C1. "Nosotros ayudamos en la cultura, no desperdiciando agua, no arrojando basuras y regando las plantas diariamente para contribuir con su desarrollo".

- Tendencia campaña: En esta tendencia tres grupos (50\%), registraron que las campañas ambientales sirven como estrategia para el fortalecimiento de la cultura ambiental en la institución.

G3.C1 "Nosotros aportamos a la cultura ambiental en nuestro colegio haciendo campañas para el mejoramiento y cuidado de las plantas y todo lo relacionado con el ambiente porque queremos marcar la diferencia en nuestro colegio".

- Tendencia reciclaje-cuidado: En esta tendencia un grupo (16,6\%), reconocieron actividades de reciclaje hace parte de las estrategias para el fortalecimiento de la cultura ambiental en la institución.

G2.C1 "En nuestro colegio podemos aportar grandes cambios para la ayuda del medio ambiente como: reciclando, cuidado del agua, las plantas y colaborando con su buen desarrollo".

Se destacan algunos elementos importantes que circundan alrededor de la cultura ambiental, uno de ellos es el hecho que los estudiantes reconozcan su importancia tanto a nivel social, educativo y familiar.

\section{CONCLUSIONES}

La educación ambiental sigue siendo percibida por los estudiantes como una tarea más con la que deben cumplir en el esquema propuesta por la normatividad educativa colombiana y no como la oportunidad para fortalecer los procesos formativos, incentivando al estudiante $y$ al docente a la apropiación del conocimiento a partir del acercamiento a las realidades y no desde imaginarios.

La actividad ambiental que involucra a estudiantes en su comunidad educativa, fortalece el sentido de pertenencia y contribuye al fomento de una cultura ambiental dentro y fuera de la institución. Esto reduce poco a poco una de las principales necesidades actuales a nivel mundial, correspondiente al cuidado del medio ambiente.

Es conveniente liderar actividades que motiven a las directivas de las instituciones educativas participantes para que realmente se comprometan con el desarrollo de los 
Bio - grafia. Escritos sobre la Biología y su Enseñanza. ISSN 2027

Edición Extraordinaria. p.p. 317 - 326

Memorias del VIII Encuentro Nacional de Experiencias en Enseñanza de la Biología y la Educación Ambiental. III Congreso Nacional de Investigación en Enseñanza de la Biología.

proyectos y así comiencen a apoyar, impulsar y liderar desde su área de gestión los PRAE, con ello se evita que los proyectos se tornen deficientes y en consecuencia regresen al activismo ecológico.

\section{BIBLIOGRAFÍA}

BAYON, P MOREJÓN, A (2006). Cultura ambiental y la construcción de entornos de reproducción social en Cuba: un reto para el siglo 21. III Conferencia Internacional La obra de Carlos Marx y los desafíos del Siglo XXI. La Habana. Cuba.

FLOREZ, G,A (2012). Saberes y prácticas de la educación ambiental en los escenarios escolares del municipio de Nobsa - Boyacá. (Tesis de maestría). Universidad Pedagógica y Tecnológica de Colombia.

LÓPEZ G., MARTA \& J. G. MORCILLO O. (2007). Las TIC en la enseñanza de la Biología en la educación secundaria: los laboratorios virtuales. Revista electrónica de la enseñanza de las Ciencias. 6 (3): 15 pp.

REIGOTA, M. (1990). Les représentations sociales de l'environnement et les pratiques pédagogiques quotidiennes des professeurs de Sciences a São Paulo-Brésil. (Tese de doutorado em pedagogia da biología), Louvain-laNeuve, Université Catholique de Louvain, Belgique.

VASILACHIS, I. (2006). Estrategias de investigación Cualitativa. Barcelona, España: Gedisa Ediciones. 